\section{The estimation of approximate sample size requirements necessary for clinical and epidemiological studies in vision sciences}

\begin{abstract}
Purpose The aim of this paper is to provide an overview of sample size estimations for the most frequent type of group studies that result in continuous, binary and ordered categorical outcomes.

Methods The theory behind power and sample size calculations is explained using the basic probability concepts that underpin the most frequently used statistical significance tests.

Results Simple formulae and tables are presented for the estimation of sample sizes necessary for efficient and effective clinical and epidemiological trials. These may be used without recourse to sophisticated and complex computer software packages. Mathematical complexity is kept to a minimum. Examples and applications from the vision sciences are specifically highlighted.

Conclusions The paper highlights, with practical examples, the concepts and computations necessary to make sample size estimations accessible to all eye professionals involved in research, diagnostic and statutory work.
\end{abstract}

Eye (2009) 23, 1589-1597; doi:10.1038/eye.2009.105; published online 15 May 2009

Keywords: power; sample size; ethics; odds ratio; ordinal data; clinical difference

\section{Introduction}

In recent years, Research Ethics Committees have paid increasing attention to the numbers required for the conduct of efficient research proposals from universities, official government research institutes, and private industrial
EA Goodall'1, J Moore ${ }^{2}$ and T Moore

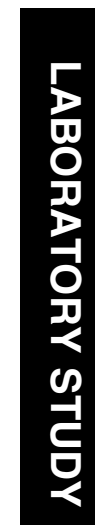

organisations. Grant proposal forms also frequently include a section on the justification for sample size numbers proposed for clinical and epidemiological trials. Many research publications incorporating quantitative and qualitative data also demand consideration of appropriate sample sizes for adequate analysis and reporting.

A substantive number of papers ${ }^{1-5}$ have been published with the objective of elucidating the calculations required for sample size estimation. The quoted references form a small proportion of the literature on the subject. Such work describes the basis of the necessary computations that rely on the fundamental concepts of probability, statistical significance, clinically meaningful differences, and the subtle concept of statistical power, the probability of detecting such differences. The latter is intimately and intricately linked to sample size estimation.

Although the subject area has been comprehensively dealt with in many research applications, there has tended to be a deficit of attention paid to it in some vision science research. This paper has the objective of elucidating the underlying concepts of power calculations and their specific application to vision science. Examples of these will be provided along with tables and formulae, which should prove to be of benefit to all eye-related professionals in the conduct of their research. Three main types of data will be considered, namely continuous, binary, and ordered categorical data. This paper will focus on studies in which two independent groups are to be compared. A second paper will concentrate on more sophisticated trials in which dependent groups are to be compared.
${ }^{1}$ Department of Statistics and Operational Research, School of Mathematics and Physics, The Queen's University of Belfast, Belfast, Northern Ireland, UK

${ }^{2}$ Mater Hospital, Belfast Health and Social Care Trust, Belfast, Northern Ireland, UK

${ }^{3}$ Centre for Molecular Biosciences, The University of Ulster, Northern Ireland, UK

Correspondence:

EA Goodall, School of Mathematics and Physics, The Queen's University of Belfast, Belfast BT7 1NN, Tel: + 4402890776580 Fax: + 4402870324375

E-mail: edwardagoodall@ hotmail.com

Received: 4 September 2008

Accepted in revised form: 11 April 2009 Published online: 15 May 2009

Proprietary interest: None Northern Ireland, UK 


\section{Materials and methods}

\section{Basic assumptions}

It is assumed that researchers who are required to perform a power calculation are familiar with the basic measures of descriptive statistics such as mean (or average), median (or middle value), and standard deviation. The former two are used to summarise a set of data and are often referred to as measures of central tendency. The latter statistic provides an estimate of how closely (or not) data are spread about the mean. A combination of the two is frequently used to characterise a set of data that follows a normal distribution in which the mean and median approximately coincide. In this case, a graphical summary of the data is bell shaped. For such a distribution, about $95 \%$ of data are contained within 1.96 standard deviations of the mean. In the comparison of a set of means (each with standard deviation $s$, and based on $n$ values each), the variance between means is reduced by a factor of $n$. The associated standard deviation, called the standard error, is estimated by $s / \sqrt{n}$. For a normal distribution of means, $\sim 95 \%$ should be contained within 1.96 standard errors of the overall mean. The boundaries of the relevant interval are called confidence limits or critical values.

Our next assumption is that researchers are acquainted with the basic notions of probability theory and, in particular, that the probability, $p$, of an event outcome lies between 0 and 1 . There are many excellent texts on basic statistical theory. For revision purposes and the use of statistical software, Goodall ${ }^{6}$ provides an introduction that focuses on applications in biomedical science without straying too far into the underpinning mathematical complexity.

\section{Statistical significance}

The concept of probability lies at the heart of statistical significance tests. It may be worthwhile to delineate the basic theory in which textbooks often use the simple experiment of tossing a coin. Such a trial has two possible outcomes, a head or a tail. If we tossed a coin 10 times and obtained 10 heads, we might ask ourselves whether the coin was really fair (that is, the probability of obtaining a head was the same as the probability of obtaining a tail and both were equal to 0.5). We might now think after carrying out our experiment that the coin was biased towards heads and that the probability of obtaining a head was higher than 0.5 .

The above situation is similar to what happens in the design and analysis of clinical trials in a variety of applications in biomedicine. We have a hypothesis that we wish to test, for example, is one drug treatment really more effective than another? A hypothesis called the null hypothesis (often abbreviated to $\mathrm{H}_{0}$ ) is set up that there is no difference. We then proceed to test this hypothesis by conducting an experiment to assess the impact of the drug on two sets of patients. If there is a major difference between the average results for each set, we would suspect that the null hypothesis is incorrect and that the alternative hypothesis, $\left(\mathrm{H}_{\mathrm{A}}\right.$, one drug is superior) is true. However, it is often difficult to know where to draw the line in making a decision. This is where probability theory is important. In the coin tossing experiment, the probability of obtaining the observed result is $(1 / 2)^{10} \ldots(1 / 1024)$ that is, $<0.001$ if the null hypothesis is true $(P<0.001)$. In this case, we would tend to favour the alternative hypothesis because the observed result was so unusual and unlikely. A scientist would now say that the results are very highly significant and reject the null hypothesis. A convention has grown up that if $P<0.05$, the results are said to be significant. With $P<0.01$, the results are said to be highly significant. Many favour the strategy of simply stating the probability of obtaining the experimental results found and allowing readers of their report to make up their own minds.

\section{Summary}

The null hypothesis $\left(\mathrm{H}_{0}\right)$ is that which we are happy to accept in the absence of any definite evidence to the contrary. It should say something precise about the population. Typically, it might say that the population is as it is claimed to be, or that the population has not changed.

The alternative hypothesis $\left(\mathrm{H}_{\mathrm{A}}\right)$ is that for which we are seeking evidence. We shall accept it only if the evidence is reasonably conclusive.

We devise some rule so that, on the basis of the evidence available, we can make a decision either to accept $\mathrm{H}_{0}$ or to reject $\mathrm{H}_{0}$. We might make the correct decision (to accept $\mathrm{H}_{0}$ when it is actually true, or to reject $\mathrm{H}_{0}$ when it is actually false) but there are two possible types of error.

To reject $\mathrm{H}_{0}$ when it is actually true.

This is called Type 1 error.

The probability of making this error is often denoted by $\alpha$, and is called the significance level of the test.

To accept $\mathrm{H}_{0}$ when it is actually false.

This is called Type 2 error.

The probability of making this error is often denoted by $\beta$.

The power of the test is $(1-\beta)$ that is, the probability of rejecting $\mathrm{H}_{0}$ when it is false. 
The tests that we shall consider are designed so as to have a given level of significance $(P<0.05)$. Power should be at least $80 \%$, preferably $90 \%$. It is closely connected to the sample size of an experiment or survey.

If $\mathrm{H}_{0}$ is rejected, this is a positive result. We can be reasonably confident that the alternative hypothesis $\mathrm{H}_{\mathrm{A}}$ is true.

If $\mathrm{H}_{0}$ is accepted, this does not mean that we are confident that $\mathrm{H}_{0}$ is true. It simply means that the null hypothesis $\mathrm{H}_{0}$ is plausible in the light of the evidence available, and cannot confidently be rejected.

\section{Clinically important difference}

A subject of confusion to some researchers is the definition of an outcome difference that is considered to be of practical importance. This is often confused with a statistically significant difference. However, the two are quite different. In fact, the most important consideration in the estimation of sample size is a realistic assessment of the minimum level of difference between two groups that it would be worthwhile to detect. Researchers should spend some time thinking about this at the beginning of their work as it can have a dramatic impact on the sample size numbers required. Well-designed research work should result in the detection of an important difference (assuming it actually exists) between groups that is also statistically significant that is, it incorporated a large enough sample size to be reasonably certain that the difference is not purely due to chance.

\section{Tests for statistical significance}

A number of standard tests have been developed to assess statistical significance between two groups of data. These are based on the theory discussed earlier. The tests vary depending on the type of data under investigation. They may be summarized as follows:(1) continuous, (2) binary, and (3) ordered categorical. It may be useful to review the main ones in current use.

\section{$\mathrm{t}$-test}

In a two-group comparative study in which the outcome measure is a continuous variable that is approximately normally distributed (for example, blood pressure or reduction in level of astigmatism), the two sample $t$-test is the usual test of choice for the analysis of results. The appropriate test statistic is calculated as follows

$$
t=\frac{\left(\bar{X}_{1}-\bar{X}_{2}\right)}{s \sqrt{\frac{2}{n}}}
$$

The numerator estimates the true difference between the means of the two groups under investigation and the denominator is its standard error. The number in each group is $n$ and $s$ is obtained by taking the square root of the average of the two sample standard deviations squared. This formula assumes that there are equal sample numbers in each group. If there are not, then the term $(2 / n)$ is replaced by $\left(\left(1 / n_{1}\right)+\left(1 / n_{2}\right)\right)$, where $n_{1}$ is the number in the first group and $n_{2}$ is the number in the second group. This situation sometimes pertains because of dropouts from a trial. The standard deviations are also appropriately weighted. However, to achieve fairness and balance, researchers usually aim to have equal numbers in each group before a trial commences. Cambridge Statistical Tables ${ }^{7}$ provide critical values (at 5\% $(P<0.05), 1 \%(P<0.01)$, and $0.1 \%(P<0.001)$ levels of significance) to assess whether $t$ is statistically significant. Estimates of $t$ that are larger in magnitude than the critical values indicate statistical significance (that is, the difference between the means was unlikely to have arisen by chance). Note that, with a very large value of $n$, statistical significance can be achieved for a difference in means of no clinical importance. Standard statistical packages such as SPSS (Chicago, IL, USA), Minitab (PA, USA), SAS (NC, USA) and STATA (TX, USA) will also produce the $P$-value for assessment. As discussed earlier, if the difference between means is also considered to be clinically meaningful, a researcher may have discovered something of interest for further investigation. Throughout this paper, it will be assumed that differences between means could arise in either direction and that the tests are two sided.

Binomial test and Chi-square $\left(\chi^{2}\right)$ test

A variable that can only take two values is referred to as binary. For example, an eye treatment may result in success or failure or a symptom of disease may be present or absent. Variables such as these are said to follow a binomial distribution rather than a normal distribution. In the comparison of the two different eye treatments, the proportion of successes, $(p)$, in each group is calculated and the two proportions compared using a binomial test. The philosophy is the same as in the earlier section with the test statistic calculated as follows

$$
z=\frac{\left(p_{\mathrm{A}}-p_{\mathrm{B}}\right)}{s \sqrt{\frac{2}{n}}}
$$

The numerator estimates the true difference between the two proportions and the denominator is its standard error. The latter may be estimated by calculating the average of the two proportions, $p$, and letting $s=\sqrt{p(1-p)}$ (Appendix 1). (Cambridge Statistical Tables $^{7}$ again provide the relevant critical value). The 
assessment of whether the difference between the two proportions arose by chance (or not) is also frequently performed using a Chi-square $\left(\chi^{2}\right)$ test that provides approximately the same $P$-value to reach a decision on statistical significance. Computer packages such as SPSS and Minitab also produce the correct $P$-value. Results are often expressed as odds ratios (OR). These are calculated by computing the odds of success for each of the two groups that is, the proportion of successes is divided by the proportion of failures. The smaller odds is then usually divided into the larger one, enabling a researcher to make a statement such as 'the odds of success for one eye treatment was three times that of the other'. This approach proves to be particularly useful when more than two proportions are to be compared simultaneously, for example, three levels of application of a drug treatment. In these trials, the logarithm of OR turns out to be more mathematically tractable. This statistic is frequently used in observational studies in which a risk factor is under investigation in a case-control study. A classic case is the linkage of smoking to lung cancer.

\section{Mann-Whitney U-test}

Many research studies result in an outcome measure that is defined on an ordered categorical scale. For example, a patient's subjective response to whether a surgical eye treatment was successful (or not) might be assessed using a Likert scale (strongly agree (1), agree (2), disagree (3), and strongly disagree (4)). In this situation, where two groups are to be compared, the usual test of choice is the Mann-Whitney $U$-test with allowance made for ties. ${ }^{8}$ This is a distribution-free test that compares the sums of ranks and usually attempts to answer the question 'Are the medians of the two groups statistically significantly different?' (see Appendix 2 for more detail).

\section{Power analyses}

Suppose that we have a two-group comparative study in which the response is measured on a continuous scale. Further, we wish the sample size in each group to be sufficiently large to be $90 \%$ certain (power $=0.90$ ) that a clinically important difference, $d$, will be detected at the $5 \%(P<0.05)$ level of significance. The appropriate test statistic is given by equation (1). Assuming a normal distribution, $t$ needs to be at least 1.96 to achieve significance. The least difference between the two means (LSD) that will be statistically significant is given by rewriting equation (1) as

$$
\mathrm{LSD}=1.96 \times s \sqrt{\frac{2}{n}}
$$

However, to be $90 \%$ certain of detecting a difference, $d$ (that is, the clinically important difference defined earlier), we must also satisfy a second equation

$$
\frac{\mathrm{LSD}-d}{s \sqrt{\frac{2}{n}}}=-1.28
$$

The value 1.28 is the critical point corresponding to the ninetieth percentile of a normal distribution. Rewriting equation (4) we have

$$
\mathrm{LSD}=d-1.28 s \sqrt{\frac{2}{n}}
$$

Equating the right-hand sides of (3) and (5), then solving for $n$, we obtain

$$
\begin{aligned}
n & =\frac{2(1.96+1.28)^{2} s^{2}}{d^{2}} \\
& \doteq 21\left(\frac{s}{d}\right)^{2}
\end{aligned}
$$

For $80 \%$ power, the 1.28 value reduces to 0.84 , the eightieth percentile of a normal distribution, and

$$
n \doteq 16\left(\frac{s}{d}\right)^{2}
$$

We can see that the lower power leads to a reduced sample size requirement. Also note that, for $90 \%$ power, if $s=d$ then we need 21 samples in each group. However, if the standard deviation, $s$, is twice the clinically important difference, $d$, then the sample size for each group increases dramatically to 84 . For $80 \%$ power, the corresponding numbers required in each group are 16 and 64 . Before a trial commences, $s$ may be estimated from an earlier pilot study or from the research literature.

For binary data, where two proportions, $p_{\mathrm{A}}$ and $p_{\mathrm{B}}$, are to be compared, first calculate $p=\left(p_{\mathrm{A}}+p_{\mathrm{B}}\right) / 2$. If this value is not too small (greater than 0.10 say), estimate $s$ by $\sqrt{p(1-p)}$ as in Section 2 and insert it into (6) and (7) to estimate the approximate sample sizes required in each group. Recall also that the clinically important difference, $d$, is defined by the researcher before the trial begins. For binary data, the numbers required are usually much higher than for continuous data. For example, if $p_{\mathrm{A}}=0.5$ and $d=0.1, p$ may be computed to be 0.55 and thus $s=0.50$.

For $90 \%$ power,

$$
\begin{aligned}
n & =21\left(\frac{0.5}{0.1}\right)^{2} \\
& =525 \text { in each group }
\end{aligned}
$$

For $80 \%$ power, $n=400$. These are slight overestimates as we are making assumptions that underlying normal distributions exist. If we wish to be more exact, correction 
factors can be applied in both the continuous and binary cases. Tables 1 and 2 provide corrected values. The simple estimates provided above do not differ too radically from the latter and various scenarios can be investigated using only a calculator.

When the outcome or response variable from a trial is measured on an ordered categorical scale (for example, 1 , $2,3,4$, and 5), the power calculation for the appropriate Mann-Whitney $U$-test is not as straightforward as in the earlier two sections. However, by making a number of reasonable assumptions, we can arrive at a good approximation of the sample sizes required in each independent group.

The mathematics involved uses the concept of the OR to estimate the clinically meaningful difference, which the researcher thinks it is important to detect. As a guideline, if an eye treatment usually results in $40 \%$ success and researchers wish to use a new treatment in which a $30 \%$ superiority of $70 \%$ is claimed, they seek an OR of

$$
\left[\frac{\left(\frac{0.7}{0.3}\right)}{\left(\frac{0.4}{0.6}\right)}\right]=3.50
$$

For one of the groups, the proportion of cases expected in each category of the scale has also to be specified. If we consider the earlier example with five categories, it is usually reasonable to assume that the mean proportions in each category are approximately equal and that the

Table 1 Sample sizes required per group at the two-sided 5\% significance level for different values of $(s / d)$ and power

\begin{tabular}{lrrrr}
\hline s/d $)$ & \multicolumn{4}{c}{ Power $(1-\beta)$} \\
\cline { 2 - 5 } & 95 & 90 & 80 & 50 \\
\hline 2.50 & 164 & 133 & 100 & 49 \\
2.00 & 105 & 86 & 64 & 32 \\
1.50 & 70 & 48 & 36 & 18 \\
1.25 & 42 & 34 & 26 & 13 \\
1.00 & 27 & 22 & 17 & 9 \\
0.50 & 9 & 6 & 5 & 4 \\
\hline
\end{tabular}

clinically meaningful difference, $d$, will be consistent. If we have two groups called $\mathrm{A}$ and $\mathrm{B}$, then denote the proportions expected in group $\mathrm{A}$ by $p \mathrm{~A} 1, p \mathrm{~A} 2, p \mathrm{~A} 3, p \mathrm{~A} 4$, and $p \mathrm{~A} 5$ with similar nomenclature for group $\mathrm{B}$. If we denote the cumulative probabilities by CA1, CA2, CA3, $\mathrm{CA} 4$, and $\mathrm{CA} 5$, then $\mathrm{CA} 1=p \mathrm{~A} 1, \mathrm{CA} 2=(p \mathrm{~A} 1+p \mathrm{~A} 2)$, and so on. The OR is the probability of a patient being in a given category or lower in one group compared with the other. For category 1, it is estimated by

$$
\text { OR1 }=\frac{\left[\left(\frac{\mathrm{CA} 1}{(1-\mathrm{CA} 1)}\right)\right]}{\left[\left(\frac{\mathrm{CB} 1}{1-\mathrm{CB} 1}\right)\right]}
$$

OR2, OR3, and OR4 can be similarly calculated. The assumption that these ORs are all approximately equal justifies the use of the Mann-Whitney $U$-test as the best one to use. The combination of this assumption with the earlier assumption that the mean proportions in each group are roughly equal leads to a formula for the numbers required in each group.

For $90 \%$ power, this is given by

$$
n=\frac{6[1.96+1.28]^{2}}{\left[\log _{e} \mathrm{OR}\right]^{2}}
$$

For $80 \%$ power, the 1.28 reduces to 0.84 . As usual, we have assumed a $5 \%$ level of statistical significance. If we assume an OR of 2 , then $\log _{e} 2.0=0.693$. For $90 \%$ power, we require $\left((6 \times 10.5) /(0.693)^{2}\right)=131$ in each group. For $80 \%$ power, 98 in each group are required.

The above estimate of the number, $n$, required in each group is approximate. A correction factor should be applied where the number of categories is small. The estimated $n$ should be multiplied by $\left(1-\left(1 / k^{2}\right)\right)^{-1}$, where $k$ is the number of categories. It is not really necessary when $k$ is greater than five. For $k=2,3,4$, and 5 , it is easily shown that the correction factors are 1.333, 1.125, 1.067 , and 1.042, respectively. Whitehead ${ }^{4}$ has shown that there is only a relatively small increase in power to be

\begin{tabular}{|c|c|c|c|c|c|c|c|c|c|c|c|c|c|c|c|c|}
\hline \multicolumn{17}{|c|}{$p B$} \\
\hline$p A$ & 0.15 & 0.20 & 0.25 & 0.30 & 0.35 & 0.40 & 0.45 & 0.50 & 0.55 & 0.60 & 0.65 & 0.70 & 0.75 & 0.80 & 0.85 & 0.90 \\
\hline 0.10 & 686 & 199 & 100 & 62 & 43 & 32 & 25 & 20 & 16 & 14 & 11 & 10 & 8 & 7 & 6 & 5 \\
\hline 0.15 & & 906 & 250 & 121 & 73 & 49 & 36 & 27 & 22 & 17 & 14 & 12 & 10 & 8 & 7 & 6 \\
\hline 0.20 & & & 1094 & 294 & 138 & 82 & 54 & 39 & 29 & 23 & 18 & 15 & 12 & 10 & 8 & 7 \\
\hline 0.25 & & & & 1251 & 329 & 152 & 89 & 58 & 41 & 31 & 24 & 19 & 15 & 12 & 10 & 8 \\
\hline 0.30 & & & & & 1377 & 356 & 163 & 93 & 61 & 42 & 31 & 24 & 19 & 15 & 12 & 10 \\
\hline 0.35 & & & & & & 1471 & 376 & 170 & 96 & 62 & 43 & 31 & 24 & 18 & 14 & 11 \\
\hline 0.40 & & & & & & & 1534 & 388 & 173 & 97 & 62 & 42 & 31 & 23 & 17 & 14 \\
\hline 0.45 & & & & & & & & 1565 & 392 & 173 & 96 & 61 & 41 & 29 & 22 & 16 \\
\hline
\end{tabular}
obtained by increasing the number of categories beyond

Table 2 Sample sizes to detect a difference in two proportions, $\mathrm{pA}$ and $\mathrm{pB}$, at a $5 \%$ significance level with $80 \%$ power 
Table 3 Numbers required in each group of ordered categorical data (for five categories) and for varying odds ratios and powers

\begin{tabular}{lrrrr}
\hline Odds ratio & \multicolumn{4}{c}{ Power } \\
\cline { 2 - 5 } & 50 & 80 & 90 & 95 \\
\hline 1.5 & 146 & 299 & 400 & 494 \\
2 & 50 & 102 & 137 & 169 \\
3 & 20 & 41 & 54 & 67 \\
4 & 13 & 26 & 34 & 43 \\
5 & 9 & 19 & 25 & 31 \\
10 & 5 & 9 & 12 & 15 \\
\hline
\end{tabular}

five. Thus, any saving in the number of patients to be recruited is minimal. Table 3 provides the numbers required for different powers and ORs when the number of categories chosen is five.

A special case of the ordered categorical data scenario is when the number of categories is equal to two. The situation here is much the same as for binary data analysis. Suppose, for example, that researchers wish to show that a new eye treatment increases success rates from 40 to $60 \%$, corresponding to an OR of 2.24 . For $80 \%$ power and $5 \%$ significance, the numbers required in each group, using the computation of this section, is given by

$$
n=\frac{(1.333) \times(47)}{0.65}=96
$$

This number is approximately the same as if the analysis had been performed using the binomial test for comparing two proportions.

\section{Results and examples}

\section{Continuous data}

In a paper by Wong et al, ${ }^{9}$ the authors evaluated the difference in the duration and power of phacoemulsification required between the in situ fracture variation of divide and conquer (DC) nucleofractis as described by Shepherd ${ }^{10}$ and the phaco chop (PC) technique, a variation of divide and conquer nucleofractis in which nuclei are divided into fragments with a phaco chopper instrument without the use of central sculpting, as described by Koch. ${ }^{11}$ They found that PC required significantly less $(P<0.001)$ phaco time in minutes (mean $=1.2$, standard deviation $=0.79, n=62$ ) than $\mathrm{DC}($ mean $=2.4$, standard deviation $=0.74, n=55)$. Other researchers wish to investigate whether they can identify comparable improved outcomes and, in the preparation of their Research Proposal, are asked to perform a power calculation to assess the sample numbers required in each experimental group. They are also asked what they wish to achieve and they state that a clinically meaningful difference between the means of the two outcome groups is $0.5 \mathrm{~min}$. A standard deviation, $s$, for the computation is taken from the earlier study as 0.75 , from the square root of the weighted averages of the two reported sample standard deviations. Assuming continuous data that are approximately normally distributed, it is envisaged that the $t$-test will be used to assess the results. The sample size (n) required for each group can be estimated from the relevant method delineated earlier. Thus, for $80 \%$ power and $5 \%$ significance,

$$
n=\frac{16 \times(0.75)^{2}}{(0.50)^{2}}=36
$$

For $90 \%$ power, $n=47$. It is also prudent to allow for 'dropouts' after initial recruitment of patients. This level may be taken as around 10-15\%. Allowing for the latter and $80 \%$ power, the researchers need to seek a total recruitment size of 84 patients. The $(d / s)$ value, in this case $((0.5 / 0.75)=0.67)$, is usually referred to as the standardised difference. Some Ethics Committees and Research Grant fund holders may wish to see several scenarios presented, that is, varying standardised difference ratios with a range of clinically meaningful differences, $d$, being defined.

\section{Binary data}

Our next example will use a paper by Sullivan et al. ${ }^{12}$ The purpose of the research was to evaluate the imaging characteristics of a cohort of patients with ocular adnexal lymphoproliferative disease (OALD). One of the major results reported was that positron emission tomography (PET) upstaged $71 \%$ of patients with systemic lymphoproliferative involvement, having a higher sensitivity than computed tomography (CT) in detecting distant disease ( 86 vs $72 \%$ ). In statistical studies, sensitivity is defined as the proportion of true positives correctly identified by a clinical test or procedure.

Many studies require the comparison of two sensitivities, such as the one quoted above, in which the outcomes are essentially binary in nature. Suppose that other researchers want to conduct a similar type of trial, perhaps with an enhancement of the earlier procedures used, and proceed to define a clinically meaningful difference between the two relevant proportions as being of the order of $20 \%$. The null hypothesis is stated that both procedures yield sensitivities of $70 \%$ and the alternative hypothesis is that there is a $20 \%$ difference between the two. They are allowing for the possibility that the difference could be in either direction (for example, two-sided) but really expect the newer 
procedure to be superior to a standard one. As before, a Research Ethics Committee demands a power calculation before approving the research. The actual test of choice here should be a variation on the $\chi^{2}$ test called McNemar's test, ${ }^{13}$ as it is planned to assess two procedures on the same set of patients. This involves the analysis of correlated data and will be dealt with in a future paper. For the purposes of this example, it will be assumed that we wish to compare a proportion of 0.7 with one that may be 0.2 better (or worse) using a straightforward test of proportions or $\chi^{2}$ test. In this case, $d=0.20$ and $s=\sqrt{p(1-p)}$ where $p$ can be taken as about 0.8 (that is, $p=(0.7+0.9) / 2$ ) and thus $s=0.40$. For a power of $80 \%$, the sample size required for each group is estimated by

$$
n=16 \frac{(0.4)^{2}}{(0.2)^{2}}=64
$$

For $90 \%$ power, $n=84$. Allow for 'dropouts' as before and the researchers should satisfy an Ethics Committee.

If the difference between two procedures is suspected to be in the other direction (for example, 0.7 vs 0.5 ), the sample size for each group will need to be considerably higher as $p$ will now equal 0.60 and $s$ will be 0.49 . For a power of $80 \%$, the sample size for each group is then given by

$$
n=16 \frac{(0.49)^{2}}{(0.2)^{2}}=96
$$

Another example is provided by the Macular Photocoagulation Study. ${ }^{14}$ In research work on agerelated macular degeneration, $0.58(98 / 169)$ of an untreated group and $0.49(86 / 174)$ of a group treated with krypton photocoagulation had lost six or more lines of visual acuity in the study eye after 3 years of followup. If the original objective was to detect a difference of 0.10 in the two groups, with the null hypothesis that there was no difference (that is, both about 0.60 ), then for the earlier power calculation, $p=0.55$ and $s=0.50$. For a power of $80 \%$, the sample size required for each group is estimated by

$$
n=16 \frac{(0.5)^{2}}{(0.10)^{2}}=400
$$

\section{Ordered categorical data}

A research paper by McMonnies ${ }^{15}$ delineates the use of a detailed questionnaire to elucidate the diagnosis and level of severity in patients with presenting signs of 'dry eye'. This approach results in a patient being scored on an ordered categorical scale of 1-25. Suppose that a researcher wishes to initiate a study in which the scale scores will be compressed into four categories indicating the relative severity of the condition with a four corresponding to very severe, three to severe, two to moderate, and one to mild. The researcher wishes to use this scale to assess the claim of a pharmaceutical company that their new eye ointment produces improvements in treatment that are twice as good as those of a competitor. From earlier analysis of retrospective results, this claim is interpreted as meaning that the proportion of patients still recording a very severe condition after treatment will be 0.5 in one group and 0.25 in the group using the new eye ointment, equating to an expected OR in the latter's favour of about three. This assumption is extrapolated across the categories as defined earlier in our consideration of ordered categorical data. As usual, an Ethics Committee is requested to consider a Research Proposal including a power calculation to estimate the sample size required in each eye treatment group. For $80 \%$ power, this can be estimated from

$$
\begin{aligned}
n & =\frac{47}{\left(\log _{e} \mathrm{OR}\right)^{2}} \\
& =\frac{47}{\left(\log _{e} 3\right)^{2}} \\
& =\frac{47}{(1.207)} \doteq 39
\end{aligned}
$$

Multiplying by the correction factor, 1.067, for four categories, this yields a sample size of 42 per group.

The Mann-Whitney $U$-test is often used on data that are not ordered on a categorical scale (as before), but an initial analysis of continuous data has shown a significant deviation from normality. An example might be data measurements of visual acuity, which, even on the $\log$ MAR scale, may not satisfy normality assumptions. For the purposes of power calculations, it could be considered as approximately categorical in nature for example, categories correspond to best corrected visual acuities of $0-0.3,>0.3-0.6$, and so on. This approach is often taken when different types of lens are to be compared with the objective of showing that a newer type leads to significantly better improvements in eyesight. Note, however, that this strategy may lead to greater sample sizes being demanded. In general, a Mann-Whitney $U$-test is less powerful than a $t$-test, except perhaps in cases where outliers in the data exist. The former test is less sensitive to the existence of these as medians, rather than means, are being investigated.

\section{Discussion}

Awareness of the need for power calculations to estimate sample sizes has become more widespread in recent 
years. Their requirement has been increasingly demanded by the Ethics Committees of Hospitals, Universities, and by those committees formally established by regional and national government action. Research Grant fund holders also inevitably necessitate the production of a power analysis to justify expenditure on valuable recurrent and capital resources.

Although it is advisable to consult a statistician at the onset of planning of a clinical or epidemiological trial, many organisations have limited access to such a service as it is often shared by a large number of medical research staff across a wide band of disciplines. Thus, the routine availability of advice may be constricted. When collaboration is instigated, it helps if both sides can speak the language of the other. It is therefore useful if researchers have an acquaintance with the nomenclature required for good statistical design and analysis.

Power calculations require some knowledge of the meaning of statistical significance level, probability, and the concept of a clinically meaningful difference or effect size. The three main types of numerical scale used in quantitative research are continuous, binary, and ordered categorical. The efficient computation of sample size needed in a two-group comparison trial is dominated by the correct choice of scale and the relevant statistical test. In turn, the choice of test leads to the most appropriate type of power calculation.

It has been the authors' experience that there has been, in some areas of the vision sciences, minimal contact and collaboration between statisticians and researchers. This paper has attempted to bridge a gap by conducting a review of the statistical theory required for the computation of adequate sample sizes needed for research trials. It is hoped that the paper will provide guidelines and form the basis for fruitful advice to those who are engaged in ophthalmologic research but who, through no fault of their own, have had little training in the use of statistical analysis. For the sake of simplicity, the paper has concentrated on power analyses for two group comparisons in which the outcomes are assumed to be independent. A future paper will concentrate on the more complex challenges faced when groups of correlated data are to be compared (for example, different tests on the same set of patients).

Although the demand for power calculations seems unnecessarily bureaucratic to some researchers, it nevertheless presents an opportunity to think in more depth about the design of a study, its aims and objectives, and how the final statistical analysis will be conducted. Many are surprised by the actual numbers required for the efficient conduct of their research. However, this challenge inevitably arises from situations in which the standard deviation of a set of data exceeds the clinical difference which it is considered important to detect. Large effect sizes combined with a comparatively low standard deviation will, in turn, reduce the sample size required for the research.

\section{Acknowledgements}

We wish to acknowledge the seminal work of Sir Ronald Aylmer Fisher ${ }^{16}$ and his classic text on Statistical Methods for Research Workers.

\section{References}

1 Campbell MJ, Julious SA, Altman DG. Estimating sample sizes for binary, ordered categorical, and continuous outcomes in two group comparisons. Br Med J 1995; 311: 1145-1148.

2 Daly L. Confidence intervals and sample sizes: don't throw out all your old sample size tables. Br Med J 1991; 302: 333-336.

3 Lehr R. Sixteen s squared over d squared: a relation for crude sample size estimates. Stat Med 1992; 11: 1099-1102.

4 Whitehead J. Sample size calculations for ordered categorical data. Stat Med 1993; 12: 2257-2272.

5 Fayers PM, Machin D. Sample size: how many patients are necessary? Br J Cancer 1995; 72: 1-9.

6 Goodall EA. Statistics for Biomedical Science, 1st ed. Blueberry Publishing: UK, 2007.

7 Lindley DV, Scott WF. New Cambridge Statistical Tables, 2nd ed. Cambridge University Press: UK, 1984.

8 Mann HB, Whitney DR. On a test of whether one of 2 random variables is stochastically larger than the other. Ann Math Stat 1947; 18: 50-60.

9 Wong T, Hingorani M, Lee V. Phacoemulsification time and power requirements in phaco chop and divide and conquer nucleofractis technique. J Cataract Refract Surg 2000; 26(9): 1374-1378.

10 Shepherd JR. In situ fracture. J Cataract Refract Surg 1990; 16: 436-440.

11 Koch PS, Katzen LE. Stop and chop phacoemulsification. J Cataract Refract Surg 1994; 20: 566-570.

12 Sullivan TJ, Valenzuela AA. Imaging features of ocular adnexal lymphoproliferative disease. Eye 2006; 20: 1189-1195.

13 McNemar Q. Note on the sampling error of the difference between correlated proportions or percentages. Psychometrika 1947; 12: 153-157.

14 Macular Photocoagulation Study Group. Krypton laser photocoagulation for neovascular lesions of age-related macular degeneration. Results of a randomized clinical trial. Arch Ophthalmol 1990; 108(6): 816-824.

15 McMonnies CW. Key questions in a dry eye history. J Am Optom Assoc 1986; 57: 512-517.

16 Fisher RA. Statistical Methods for Research Workers, 14th ed. Oliver and Boyd Ltd: Edinburgh, 1970. 


\section{Appendix 1}

\section{Binomial distribution}

If a sterling coin is tossed, there are two outcomes, a head $(\mathrm{H})$ or a tail $(\mathrm{T})$. If the coin is fair, the probability, $p$, of each is $(1 / 2)(=0.5)$. If a coin is tossed 100 times $(n=100)$, on average, we should obtain 50 heads. Mean $=n p=$ $(100) 2)=50$. In practice, we will not obtain exactly 50 . A variable $X$ with a Binomial distribution is known to have a variance of $n p(1-p)$. The standard deviation is thus $\sqrt{n p(1-p)}$. For the above example, the standard deviation is five. For large $n$, the binomial may be approximated by a normal distribution and we can make statements such as $95 \%$ confidence limits for the mean are mean $(n p) \pm 1.96 \sqrt{n p(1-p)}$. For the above example, the limits would be approximately $40 \rightarrow 60$ [50 \pm 10$]$. If we obtained values outside these limits, we would suspect that the coin was not fair.

Suppose we are investigating the sensitivity of a test that is, proportion $p$ of test positives $(X)$ from a sample of $n$ true positives

$$
\begin{aligned}
\operatorname{variance}(p) & =\operatorname{variance}\left(\frac{X}{n}\right) \\
& =\frac{1}{n^{2}} \operatorname{variance}(X) \\
& =\frac{1}{n^{2}} n(p)(1-p) \\
& =\frac{p(1-p)}{n} \\
\text { Hence standard error } & =\sqrt{\frac{p(1-p)}{n}}
\end{aligned}
$$

\section{Appendix 2}

\section{The Mann-Whitney U-test}

This is a distribution-free ranking test that asks the question 'Are the medians of two sets of data the same?'

1. Suppose there are two samples, with values $x_{1}, x_{2}, x_{3}, \ldots, x_{N_{x}}$ and $y_{1}, y_{2}, y_{3}, \ldots, y_{N_{y}}$. Rank them in order together. This gives a sequence such as

$$
x y y x x y
$$

2. For each $x$ value, count the number of $y$ values that come after it. Thus, in the above example, the first $x$ precedes three $y$ values, the second one, the third one, and the fourth none.

3. Form the total $3+1+1+0$ and call it $U_{x}$, the number of times an $x$ precedes a $y$. In the same way, find $U_{y}$-here $3+3+1=7$. Check that

$$
U_{x}+U_{y}=N_{x} N_{y}
$$

Under the null hypothesis that the averages are the same, one expects $U_{x}=U_{y}=(1 / 2) N_{x} N_{y}$, as each $x$ value will on average have half the $y$ sample behind it and the other half in front. If the medians are significantly different, say $x$ is ahead of $y$, then the $x$ values will precede more than their fair share of $y$ values, and $U_{x}$ will be greater than $U_{y}$. For small samples, the significance is given by tables. For large samples, the normal approximation can be used, with the mean of $U_{x}$ equal to $(1 / 2) N_{x} N_{y}$, and variance $(1 / 12) N_{x} N_{y}\left(N_{x}+N_{y}\right)$. 